Homology, Homotopy and Applications, vol.15(2), 2013, pp.145-151

\title{
ON MONADS OF EXACT REFLECTIVE LOCALIZATIONS OF ABELIAN CATEGORIES
}

\author{
MOHAMED BARAKAT AND MARKUS LANGE-HEGERMANN
}

\author{
(communicated by Winfried Bruns)
}

\begin{abstract}
In this paper we define Gabriel monads as the idempotent monads associated to exact reflective localizations in Abelian categories and characterize them by a simple set of properties. The coimage of a Gabriel monad is a Serre quotient category. The Gabriel monad induces an equivalence between its coimage and its image, the localizing subcategory of local objects.
\end{abstract}

\section{Introduction}

Abelian categories were introduced in Grothendieck's Tôhoku paper [Gro57] and since then became a central notion in homological algebra. In our attempt to establish a constructive setup for homological algebra, we introduced in [BLH11, Chap. 2] the notion of a computable Abelian category, i.e., an Abelian category in which all existential quantifiers occurring in the defining axioms can be turned into algorithms. Along these lines we treated in loc. cit. the Abelian categories of finitely presented modules over so-called computable rings and their localization at certain maximal ideals.

Our next goal is to treat the Abelian category $\mathfrak{C o h} X$ of coherent sheaves on a projective scheme $X$ along the same lines. This category is, by Serre's seminal paper [Ser55], equivalent to a Serre quotient $\mathcal{A} / \mathcal{C}$ of an Abelian category $\mathcal{A}$ of graded modules over the graded ring $S=k\left[x_{0}, \ldots, x_{n}\right] / I$, where $I=I(X)$ is the homogeneous ideal defining $X$ and $\mathcal{C}$ is the thick subcategory of graded modules with zero sheafification. In the context of this paper we require the thick subcategory $\mathcal{C} \subset \mathcal{A}$ to be localizing. Indeed, there are several ways to model $\mathfrak{C o h} X$ as a Serre quotient $\mathcal{A} / \mathcal{C}$ where $\mathcal{A}$ is some Abelian category of graded $S$-modules, but not in all models is the thick subcategory $\mathcal{C} \subset \mathcal{A}$ localizing. Serre defined in loc. cit. $\mathcal{A}$ as the category of quasi finitely generated graded $S$-modules, i.e., of graded modules $M$ such that the truncated submodule $M_{\geqslant d}$ is finitely generated for some $d \in \mathbb{Z}$ large enough. Here $\mathcal{C} \subset \mathcal{A}$ is localizing but this model is not constructive. Redefining $\mathcal{A}, \mathcal{C}$ to be the respective full subcategories of finitely generated graded $S$-modules indeed yields a constructive model $\mathcal{A} / \mathcal{C} \simeq \mathfrak{C o h} X$, but now $\mathcal{C} \subset \mathcal{A}$ is no longer localizing. Luckily, for each $d_{0} \in \mathbb{Z}$ the respective full subcategories $\mathcal{A}, \mathcal{C}$ of graded $S$-modules truncated

Received January 16, 2013, revised July 28, 2013; published on November 24, 2013.

2010 Mathematics Subject Classification: 18E35, 18A40.

Key words and phrases: Serre quotient, reflective localization of Abelian categories, idempotent monad, Gabriel localization, saturating monad.

Article available at http://intlpress.com/HHA/v15/n2/a8 and doi:10.4310/HHA.2013.v15.n2.a8

Copyright (C) 2013, International Press. Permission to copy for private use granted. 
at $d_{0}$ yield a constructive model $\mathcal{A} / \mathcal{C} \simeq \mathfrak{C o h} X$ in which $\mathcal{C} \subset \mathcal{A}$ is again localizing (for more details cf. [BLH13, §4.1]). In all models, a coherent sheaf $\mathcal{F}$ is given by a class of graded modules isomorphic in high degrees. In the last model, the truncated (and hence finitely generated) module of twisted global sections $\bigoplus_{d \geqslant d_{0}} H^{0}(\mathcal{F}(d))$ is in the following sense a distinguished representative within this class; it is a so-called saturated object with respect to $\mathcal{C}$.

The appropriate categorical setup for a Serre quotient $\mathcal{A} / \mathcal{C}$ of an Abelian category $\mathcal{A}$ modulo a thick subcategory $\mathcal{C} \subset \mathcal{A}$ was introduced by Grothendieck [Gro57, Chap. 1.11] and then more elaborately in Gabriel's thesis [Gab62]. Later Gabriel and Zisman developed in [GZ67] a localization theory of categories in which a Serre quotient $\mathcal{A} / \mathcal{C}$ is an outcome of a special localization $\mathcal{A} \rightarrow \mathcal{A}\left[\Sigma^{-1}\right] \simeq \mathcal{A} / \mathcal{C}$. Their theory is also general enough to enclose Verdier's localization of triangulated categories, which he used in his 1967 thesis (cf. [Ver96]) to define derived categories. Thanks to Simpson's work [Sim06], the Gabriel-Zisman localization is now completely formalized in the proof assistant Coq [Coq04]. In many applications, as assumed in [GZ67], the localization $\mathcal{A}\left[\Sigma^{-1}\right]$ is equivalent to a full subcategory of $\mathcal{A}$, the subcategory of all $\Sigma$-local objects of $\mathcal{A}$. This favorable situation (which in our context means that $\mathcal{C}$ is a localizing subcategory of $\mathcal{A}$ ) is characterized by the existence of an idempotent monad associated to the localization. For a further overview on localizations we refer to the arXiv version of [Tho11].

In our application to $\mathfrak{C o h} X$ we are in the setup of Serre quotient categories $\mathcal{A} / \mathcal{C}$, which are the outcome of an exact localization having an associated idempotent monad. We call this monad the Gabriel monad (cf. Definition 2.3). Gabriel monads satisfy a set of properties that we use as a simple set of axioms to define what we call a $\mathcal{C}$-saturating monad.

The goal of this paper is to characterize Gabriel monads conversely as $\mathcal{C}$-saturating monads (Theorem 3.6). Such a characterization enables us in [BLHa] to show that several known algorithmically computable functors in the context of coherent sheaves on a projective scheme ${ }^{1}$ are $\mathcal{C}$-saturating. ${ }^{2}$ This yields a constructive, unified, and simple proof that those functors are equivalent to the functor $M \mapsto \bigoplus_{d \geqslant d_{0}} H^{0}(\widetilde{M}(d))$, and hence compute the truncated module of twisted global sections. Among those are the functors computing the graded ideal transform and the graded module given by the 0 -th strand of the Tate resolution.

The proof there relies on checking the defining set of axioms of a $\mathcal{C}$-saturating monad, which turns out to be a relatively easy task. In particular, the proof does not rely on the (full) BGG correspondence [BGG78] of triangulated categories, the Serre-Grothendieck correspondence [BS98, 20.3.15], or the local duality, as used in [EFS03].

A stronger computability notion is that of the Ext-computability. Furthermore, in $[\mathbf{B L H b}]$ we use the Gabriel monad of a localizing Serre quotient $\mathcal{A} / \mathcal{C}$ to show that the so-called Ext-computability of $\mathcal{A} / \mathcal{C}$ follows from that of $\mathcal{A}$. In particular, the Abelian category $\mathfrak{C o h} X$ is Ext-computable (cf. [BLHa]).

\footnotetext{
${ }^{1}$ This technique applies to other classes of varieties admitting a finitely generated Cox ring $S$.

${ }^{2}$ In this context the $\Sigma$-local objects are Gabriel's $\mathcal{C}$-saturated objects.
} 


\section{Preliminaries}

We refer to [Gab62, GZ67, Bor94a, Bor94b] or the active $n$ Lab wiki [nLa12] for further details and proofs. See also the arXiv version of this paper.

A monad is an endofunctor $\mathscr{W}: \mathcal{A} \rightarrow \mathcal{A}$ together with natural transformations $\eta: \operatorname{Id}_{\mathcal{A}} \rightarrow \mathscr{W}$ and $\mu: \mathscr{W}^{2} \rightarrow \mathscr{W}$ called the unit and multiplication of the monad, respectively, such that the two zig-zag identites hold. A monad $\mathscr{W}$ is called idempotent if its multiplication is a natural isomorphism. A full subcategory $\mathcal{B} \subset \mathcal{A}$ is called a reflective subcategory if the inclusion functor $\iota: \mathcal{B} \hookrightarrow \mathcal{A}$ has a left adjoint, called the reflector. A monad is idempotent if and only if its essential image is a reflective subcategory. A localization is called reflective if it admits a fully faithful right adjoint.

Proposition 2.1. Let $\mathscr{F} \dashv \mathscr{G}: \mathcal{B} \rightarrow \mathcal{A}$ be adjoint functors with unit $\eta$ and counit $\delta$ and let $(\mathscr{W}, \eta, \mu):=(\mathscr{G} \circ \mathscr{F}, \eta, \mathscr{F} \delta \mathscr{G})$ be the associated monad. The following are equivalent:

1. $\mathscr{G}$ is fully faithful, i.e., $\mathcal{B}$ is equivalent to its (essential) image under $\mathscr{G}$.

2. The counit $\delta: \mathscr{F} \circ \mathscr{G} \rightarrow I d_{\mathcal{B}}$ is a natural isomorphism. ${ }^{3}$

3. $\mathscr{W}$ is idempotent, i.e., the essential image of $\mathscr{G}$ is a reflective subcategory.

Then, $\mathscr{W}(\mathcal{A}) \simeq \mathscr{G}(\mathcal{B}) \simeq \mathcal{B}$ and $\mathscr{G}$ is conservative, i.e., reflects isomorphisms.

From now on $\mathcal{A}$ denotes an Abelian category. A full subcategory $\mathcal{C} \neq \emptyset$ of $\mathcal{A}$ is called thick if it is closed under passing to subobjects, factor objects, and extensions. From now on let $\mathcal{C}$ denote a thick subcategory of $\mathcal{A}$. The (Serre) quotient category $\mathcal{A} / \mathcal{C}$ is a category with the objects of $\mathcal{A}$ and Hom-groups

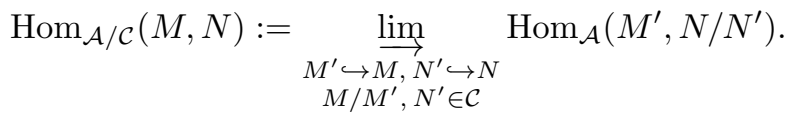

The canonical functor $\mathscr{Q}: \mathcal{A} \rightarrow \mathcal{A} / \mathcal{C}$ is defined to be the identity on objects and maps a morphism $\varphi \in \operatorname{Hom}_{\mathcal{A}}(M, N)$ to its image in the direct $\operatorname{limit} \operatorname{Hom}_{\mathcal{A} / \mathcal{C}}(M, N)$. The category $\mathcal{A} / \mathcal{C}$ is Abelian and the canonical functor $\mathscr{Q}$ is exact.

Proposition 2.2. Let $\mathscr{G}: \mathcal{A} \rightarrow \mathcal{D}$ be an exact functor into the Abelian category $\mathcal{D}$. If $\mathscr{G}(\mathcal{C})$ is zero then there exists a unique functor $\mathscr{H}: \mathcal{A} / \mathcal{C} \rightarrow \mathcal{D}$ such that $\mathscr{G}=\mathscr{H} \circ \mathscr{Q}$.

An object $M \in \mathcal{A}$ is called $\mathcal{C}$-saturated if it has no subobject in $\mathcal{C}$ and every extension of $M$ by an object $C \in \mathcal{C}$ is trivial. Denote by $\operatorname{Sat}_{\mathcal{C}}(\mathcal{A}) \subset \mathcal{A}$ the full subcategory of $\mathcal{C}$-saturated objects. We say that $\mathcal{A}$ has enough $\mathcal{C}$-saturated objects if for each $M \in \mathcal{A}$ there exists a $\mathcal{C}$-saturated object $N$ and a morphism $\eta_{M}: M \rightarrow N$ such that $\operatorname{ker} \eta_{M} \in \mathcal{C}$. The thick subcategory $\mathcal{C}$ is called localizing if the canonical functor $\mathscr{Q}$ admits a right adjoint $\mathscr{S}: \mathcal{A} / \mathcal{C} \rightarrow \mathcal{A}$, called the section functor of $\mathscr{Q}$. The category $\mathcal{C} \subset \mathcal{A}$ is localizing if and only if $\mathcal{A}$ has enough $\mathcal{C}$-saturated objects. The section functor $\mathscr{S}: \mathcal{A} / \mathcal{C} \rightarrow \mathcal{A}$ is left exact and preserves direct sums.

Definition 2.3. We call a canonical functor $\mathscr{Q}: \mathcal{A} \rightarrow \mathcal{A} / \mathcal{C}$ admitting a section functor a Gabriel localization and the associated monad $(\mathscr{S} \circ \mathscr{Q}, \eta, \mu=\mathscr{S} \delta \mathscr{Q})$ the Gabriel monad.

\footnotetext{
${ }^{3}$ In particular, $\mathscr{G}$ is a right inverse of $\mathscr{F}$ and $\mathscr{F}$ is essentially surjective.
} 
Proposition 2.4. Let $\mathcal{C}$ be a localizing subcategory of $\mathcal{A}$ with section functor $\mathscr{S}$.

1. The counit of the adjunction $\delta: \mathscr{Q} \circ \mathscr{S} \stackrel{\sim}{\rightarrow} \operatorname{Id}_{\mathcal{A} / \mathcal{C}}$ is a natural isomorphism.

2. An object $M$ in $\mathcal{A}$ is $\mathcal{C}$-saturated if and only if $\eta_{M}: M \rightarrow(\mathscr{S} \circ \mathscr{Q})(M)$ is an isomorphism, where $\eta$ is the unit of the adjunction.

3. $\mathscr{S}(\mathcal{A} / \mathcal{C}) \simeq \operatorname{Sat}_{\mathcal{C}}(\mathcal{A})$ are reflective subcategories of $\mathcal{A}$.

4. $\eta(\mathscr{S} \circ \mathscr{Q})=(\mathscr{S} \circ \mathscr{Q}) \eta$.

Corollary 2.5. In Proposition 2.4, the image $\mathscr{S}(\mathcal{A} / \mathcal{C})$ of $\mathscr{S}$ is a subcategory of $\operatorname{Sat}_{\mathcal{C}}(\mathcal{A})$ and the inclusion functor $\mathscr{S}(\mathcal{A} / \mathcal{C}) \hookrightarrow \operatorname{Sat}_{\mathcal{C}}(\mathcal{A})$ is an equivalence of categories with the restricted-corestricted Gabriel monad $\mathscr{S} \circ \mathscr{Q}: \operatorname{Sat}_{\mathcal{C}}(\mathcal{A}) \rightarrow \mathscr{S}(\mathcal{A} / \mathcal{C})$ as a quasi-inverse. In other words, $\operatorname{Sat}_{\mathcal{C}}(\mathcal{A})$ is the essential image of $\mathscr{S}$ and of the Gabriel monad $\mathscr{S} \circ \mathscr{Q}$.

Corollary 2.6. In Proposition 2.4, the restricted canonical functor $\mathscr{Q}: \operatorname{Sat}_{\mathcal{C}}(\mathcal{A}) \rightarrow$ $\mathcal{A} / \mathcal{C}$ and the corestricted section functor $\mathscr{S}: \mathcal{A} / \mathcal{C} \rightarrow \operatorname{Sat}_{\mathcal{C}}(\mathcal{A})$ are quasi-inverse equivalences of categories. In particular, $\operatorname{Sat}_{\mathcal{C}}(\mathcal{A}) \simeq \mathscr{S}(\mathcal{A} / \mathcal{C}) \simeq \mathcal{A} / \mathcal{C}$ is an Abelian category. Define the reflector $\widehat{\mathscr{Q}}$ as the corestriction of the adjunction monad $\mathscr{S} \circ \mathscr{Q}$ to its essential image $\operatorname{Sat}_{\mathcal{C}}(\mathcal{A})$, i.e., $\widehat{\mathscr{Q}}:=\operatorname{co}_{-\operatorname{res}_{\operatorname{Sat}}(\mathcal{A})}(\mathscr{S} \circ \mathscr{Q}): \mathcal{A} \rightarrow \operatorname{Sat}_{\mathcal{C}}(\mathcal{A})$. Under the above equivalence, the adjunction $\widehat{\mathscr{Q}} \dashv \iota: \operatorname{Sat}_{\mathcal{C}}(\mathcal{A}) \hookrightarrow \mathcal{A}$ corresponds to the adjunction $\mathscr{Q} \dashv \mathscr{S}: \mathcal{A} / \mathcal{C} \rightarrow \mathcal{A}$. They both share the same adjunction monad $\mathscr{S} \circ \mathscr{Q}=\iota \circ$ $\widehat{\mathscr{Q}}: \mathcal{A} \rightarrow \mathcal{A}$. In particular, the reflector $\widehat{\mathscr{Q}}$ is exact and $\iota$ is left exact.

\section{Characterizing reflective Gabriel localizations and Gabriel monads}

The next proposition states that in fact all exact reflective localizations in the setup of Abelian categories are (reflective) Gabriel localizations.

Proposition 3.1 ([Gab62, Proposition III.2.5], [GZ67, Chap. 1.2.5.d]). Let $\widetilde{\mathscr{Q}} \dashv$ $\widetilde{\mathscr{S}}: \mathcal{B} \rightarrow \mathcal{A}$ be a pair of adjoint functors of Abelian categories. Assume, that $\widetilde{\mathscr{Q}}$ is exact and the counit $\delta: \widetilde{\mathscr{Q}} \circ \widetilde{\mathscr{S}} \rightarrow \mathrm{Id}_{\mathcal{B}}$ of the adjunction is a natural isomorphism. Then $\mathcal{C}:=\operatorname{ker} \widetilde{\mathscr{Q}}$ is a localizing subcategory of $\mathcal{A}$ and the adjunction $\widetilde{\mathscr{Q}} \dashv \widetilde{\mathscr{S}}$ induces an adjoint equivalence from $\mathcal{B}$ to $\mathcal{A} / \mathcal{C}$.

Now, we approach the central definition of this paper which collects some properties of Gabriel monads.

Definition 3.2. Let $\mathcal{C} \subset \mathcal{A}$ be a localizing subcategory of the Abelian category $\mathcal{A}$ and let $\iota: \operatorname{Sat}_{\mathcal{C}}(\mathcal{A}) \hookrightarrow \mathcal{A}$ the full embedding. We call an endofunctor $\mathscr{W}: \mathcal{A} \rightarrow \mathcal{A}$ together with a natural transformation $\widetilde{\eta}: \operatorname{Id}_{\mathcal{A}} \rightarrow \mathscr{W} \mathcal{C}$-saturating if the following holds:

1. $\mathcal{C} \subset \operatorname{ker} \mathscr{W}$,

2. $\mathscr{W}(\mathcal{A}) \subset \operatorname{Sat}_{\mathcal{C}}(\mathcal{A})$

3. $\mathscr{G}:=\operatorname{co-res}_{\operatorname{Sat}_{\mathcal{C}}(\mathcal{A})} \mathscr{W}$ is exact,

4. $\widetilde{\eta} \mathscr{W}=\mathscr{W} \widetilde{\eta}$, and

5. $\widetilde{\eta} \iota: \operatorname{Id}_{\mathcal{A} \mid \operatorname{Sat}_{\mathcal{C}}(\mathcal{A})} \rightarrow \mathscr{W}_{\mid \operatorname{Sat}_{\mathcal{C}}(\mathcal{A})}$ is a natural isomorphism. ${ }^{4}$

${ }^{4}$ In particular, $\mathscr{W}(\mathcal{A})$ is an essentially wide subcategory of $\operatorname{Sat}_{\mathcal{C}}(\mathcal{A})$. 
Let $\mathscr{H}$ be the unique functor from Proposition 2.2 such that $\mathscr{G}=\mathscr{H} \circ \mathscr{Q}$. We call the composed functor $\widetilde{\mathscr{H}}:=\iota \circ \mathscr{H}$ the colift of $\mathscr{W}$ along $\mathscr{Q}$, since $\mathscr{W}=\iota \circ \mathscr{G}=\widetilde{\mathscr{H}} \circ \mathscr{Q}$.

Lemma 3.3. Let $\mathscr{Q}: \mathcal{A} \rightarrow \mathcal{A} / \mathcal{C}$ be a Gabriel localization with section functor $\mathscr{S}$. Then each $\mathcal{C}$-saturating endofunctor $\mathscr{W}$ of $\mathcal{A}$ is naturally isomorphic to $\mathscr{S} \circ \mathscr{Q}$. Furthermore, the colift $\widetilde{\mathscr{H}}$ of $\mathscr{W}$ along $\mathscr{Q}$ is also a section functor naturally isomorphic to $\mathscr{S}$.

Proof. For $\mathscr{G}:=\operatorname{co-res}_{\mathrm{Sat}_{\mathcal{C}}(\mathcal{A})} \mathscr{W}$, let $\mathscr{H}$ be the unique functor from Proposition 2.2 (using Definition 3.2.(1)) such that $\mathscr{G}=\mathscr{H} \circ \mathscr{Q}$. Then

$$
\begin{array}{rlrl}
\mathscr{G} & =\mathscr{H} \circ \mathscr{Q} & & \\
& \simeq \mathscr{H} \circ \mathscr{Q} \circ \mathscr{S} \circ \mathscr{Q} & & \left(\operatorname{Id}_{\mathcal{A} / \mathcal{C}} \simeq \mathscr{Q} \circ \mathscr{S}\right. \text { by Proposition 2.4.(1)) } \\
& =\mathscr{G} \circ \mathscr{S} \circ \mathscr{Q} & & \\
& \simeq \operatorname{Id}_{\operatorname{Sat}_{\mathcal{C}}(\mathcal{A})} \circ \mathscr{S} \circ \mathscr{Q} & \left(\operatorname{using} \mathscr{S}(\mathscr{Q}(\mathcal{A})) \subset \operatorname{Sat}_{\mathcal{C}}(\mathcal{A})\right. \text { and 3.2.(5)) } \\
& =\operatorname{co-}_{\operatorname{Sat}}(\mathcal{A}) & (\mathscr{S} \circ \mathscr{Q}) &
\end{array}
$$

and, using the notation of Definition 3.2,

$$
\mathscr{W}=\widetilde{\mathscr{H}} \circ \mathscr{Q}=\iota \circ \mathscr{G} \simeq \mathscr{S} \circ \mathscr{Q} .
$$

This also proves the equivalence $\widetilde{\mathscr{H}} \simeq \mathscr{S}$, as $\mathscr{Q}$ is surjective.

Proposition 3.4. Let $\mathscr{Q}: \mathcal{A} \rightarrow \mathcal{A} / \mathcal{C}$ be a Gabriel localization and $(\mathscr{W}, \widetilde{\eta})$ be a $\mathcal{C}$-saturating endofunctor of $\mathcal{A}$ with colift $\widetilde{\mathscr{H}}$ along $\mathscr{Q}$. Then there exists a natural transformation $\widetilde{\delta}: \mathscr{Q} \circ \widetilde{\mathscr{H}} \rightarrow \operatorname{Id}_{\mathcal{A} / \mathcal{C}}$ such that $\mathscr{Q}$ and $\widetilde{\mathscr{H}}$ form an adjoint pair $\mathscr{Q} \dashv \widetilde{\mathscr{H}}$ with unit $\widetilde{\eta}$ and counit $\widetilde{\delta}$.

Definition 3.5. Hence, each $\mathcal{C}$-saturating endofunctor $(\mathscr{W}, \widetilde{\eta})$ is the $\operatorname{monad}(\mathscr{W}, \widetilde{\eta}$, $\widetilde{\mathscr{H}} \widetilde{\delta} \mathscr{Q})$ associated to the adjunction $\mathscr{Q} \dashv \widetilde{\mathscr{H}}$. We call it a $\mathcal{C}$-saturating monad.

Proof of Proposition 3.4. We define a natural transformation $\widetilde{\delta}: \mathscr{Q} \circ \widetilde{\mathscr{H}} \rightarrow \operatorname{Id}_{\mathcal{A} / \mathcal{C}}$ and show the two zig-zag identities, i.e., that the compositions of natural transformations

$$
\mathscr{Q} \stackrel{2 \widetilde{\eta}}{\longrightarrow} \mathscr{Q} \circ \widetilde{\mathscr{H}} \circ \mathscr{Q} \stackrel{\widetilde{\delta} \mathscr{Q}}{\longrightarrow} \mathscr{Q} \text { and } \widetilde{\mathscr{H}} \stackrel{\widetilde{\eta} \mathscr{\mathscr { H }}}{\longrightarrow} \widetilde{\mathscr{H}} \circ \mathscr{Q} \circ \widetilde{\mathscr{H}} \stackrel{\widetilde{\mathscr{H}}}{\longrightarrow} \widetilde{\mathscr{H}}
$$

are the identity of functors. By 3.2.(5) we know that $(\widetilde{\mathscr{H}} \circ \mathscr{Q}) \widetilde{\eta}=\mathscr{W} \widetilde{\eta} \stackrel{3.2 .(4)}{=} \widetilde{\eta} \mathscr{W}=$ $(\widetilde{\eta} \iota) \mathscr{G}$ is an isomorphism. Hence, also $\mathscr{Q} \widetilde{\eta}$ is a natural isomorphism, because the functor $\widetilde{\mathscr{H}}$ is equivalent to $\mathscr{S}$ by Lemma 3.3 and, thus, reflects isomorphisms. This allows us to define $\widetilde{\delta}$ in such a way to satisfy the first zig-zag identity, i.e., set $\widetilde{\delta} \mathscr{Q}:=$ $(\mathscr{Q} \widetilde{\eta})^{-1}$. This defines $\widetilde{\delta}$ as $\mathscr{Q}$ is surjective (on objects). The second zig-zag identity is equivalent, again due to the surjectivity of $\mathscr{Q}$, to the second zig-zag identity applied to $\mathscr{Q}$, i.e., $(\widetilde{\mathscr{H}} \widetilde{\delta} \mathscr{Q}) \circ \widetilde{\eta}(\widetilde{\mathscr{H}} \circ \mathscr{Q})$ being the identity transformation of the functor $\widetilde{\mathscr{H}} \circ$ $\mathscr{Q}=\mathscr{W}$. Now

$$
\begin{aligned}
(\widetilde{\mathscr{H}} \tilde{\delta} \mathscr{Q}) \circ \widetilde{\eta}(\widetilde{\mathscr{H}} \circ \mathscr{Q}) & =\left(\widetilde{\mathscr{H}}(\mathscr{Q} \widetilde{\eta})^{-1}\right) \circ \widetilde{\eta}(\widetilde{\mathscr{H}} \circ \mathscr{Q}) \quad\left(\text { by the definition } \widetilde{\delta} \mathscr{Q}:=(\mathscr{Q} \widetilde{\eta})^{-1}\right) \\
& =((\widetilde{\mathscr{H}} \circ \mathscr{Q}) \widetilde{\eta})^{-1} \circ \widetilde{\eta}(\widetilde{\mathscr{H}} \circ \mathscr{Q}) \\
& =(\widetilde{\eta}(\widetilde{\mathscr{H}} \circ \mathscr{Q}))^{-1} \circ \widetilde{\eta}(\widetilde{\mathscr{H}} \circ \mathscr{Q}) \quad(\text { by } \widetilde{\eta}(\widetilde{\mathscr{H}} \circ \mathscr{Q}) \stackrel{3.2 .(4)}{=}(\widetilde{\mathscr{H}} \circ \mathscr{Q}) \widetilde{\eta}) \\
& =\operatorname{Id}_{\widetilde{\mathscr{H}} \circ \mathscr{Q}} .
\end{aligned}
$$


We now approach our main result.

Theorem 3.6 (Characterization of Gabriel monads). Each Gabriel monad is a $\mathcal{C}$ saturating monad. Conversely, each $\mathcal{C}$-saturating monad is equivalent to a Gabriel monad.

Proof. The conditions in Definition 3.2 clearly apply to a Gabriel monad $\mathscr{S} \circ \mathscr{Q}$ by definition of the canonical functor $\mathscr{Q}$, Corollary 2.5, Corollary 2.6, Proposition 2.4(4), and Proposition 2.4(2), respectively.

The converse follows directly from Lemma 3.3 and Proposition 3.4 which prove that the two adjunctions $\mathscr{Q} \dashv \widetilde{\mathscr{H}}$ and $\mathscr{Q} \dashv \mathscr{S}$ are equivalent and so are their associated monads.

\section{Acknowledgments}

We would like to thank the anonymous referee for many valuable suggestions.

\section{References}

[BGG78] I. N. Bernšteřn, I. M. Gel'fand, and S. I. Gel'fand, Algebraic vector bundles on $\mathbf{P}^{n}$ and problems of linear algebra, Funktsional. Anal. i Prilozhen. 12 (1978), no. 3, 66-67. MR 509387 (80c:14010a)

[BLHa] M. Barakat and M. Lange-Hegermann, A constructive approach to coherent sheaf cohomology via Gabriel monads, in preparation.

[BLHb] M. Barakat and M. Lange-Hegermann, On the Ext-computability of Serre quotient categories, submitted, arXiv:1212.4068.

[BLH11] M. Barakat and M. Lange-Hegermann, An axiomatic setup for algorithmic homological algebra and an alternative approach to localization, J. Algebra Appl. 10 (2011), no. 2, 269-293, arXiv:1003.1943. MR 2795737 (2012f:18022)

[BLH13] M. Barakat and M. Lange-Hegermann, Characterizing Serre quotients with no section functor and applications to coherent sheaves, Appl. Categor. Struct. (2013), published online, arXiv:1210.1425.

[Bor94a] F. Borceux, Handbook of categorical algebra. 1. Basic category theory, Encyclopedia of Mathematics and its Applications, vol. 50, Cambridge University Press, Cambridge, 1994. MR 1291599 (96g:18001a)

[Bor94b] F. Borceux, Handbook of categorical algebra. 2. Categories and structures, Encyclopedia of Mathematics and its Applications, vol. 51, Cambridge University Press, Cambridge, 1994. MR 1313497 (96g:18001b)

[BS98] M. P. Brodmann and R. Y. Sharp, Local cohomology: an algebraic introduction with geometric applications, Cambridge Studies in Advanced Mathematics, vol. 60, Cambridge University Press, Cambridge, 1998. MR 1613627 (99h:13020)

[Coq04] Coq development team, The coq proof assistant reference manual, LogiCal Project, 2004, Version 8.0. 
[EFS03] D. Eisenbud, G. Fløystad, and F.-O. Schreyer, Sheaf cohomology and free resolutions over exterior algebras, Trans. Amer. Math. Soc. 355 (2003), no. 11, 4397-4426 (electronic). MR 1990756 (2004f:14031)

[Gab62] P. Gabriel, Des catégories abéliennes, Bull. Soc. Math. France 90 (1962), 323-448. MR 0232821 (38 \#1144)

[Gro57] A. Grothendieck, Sur quelques points d'algèbre homologique, Tôhoku Math. J. (2) 9 (1957), 119-221. MR 0102537 (21 \#1328)

[GZ67] P. Gabriel and M. Zisman, Calculus of fractions and homotopy theory, Ergebnisse der Mathematik und ihrer Grenzgebiete, Band 35, SpringerVerlag New York, Inc., New York, 1967. MR 0210125 (35 \#1019)

[nLa12] nLab authors, The nlab, 2012, http://ncatlab.org/nlab/ [Online; accessed 10-February-2012].

[Ser55] J.-P. Serre, Faisceaux algébriques cohérents, Ann. of Math. (2) 61 (1955), 197-278. MR 0068874 (16,953c)

[Sim06] C. Simpson, Explaining Gabriel-Zisman localization to the computer, J. Automat. Reason. 36 (2006), no. 3, 259-285. MR 2288803 (2007h:68176)

[Tho11] S. Thomas, On the 3-arrow calculus for homotopy categories, Homology Homotopy Appl. 13 (2011), no. 1, 89-119, arXiv:1001.4536. MR 2803869

[Ver96] J.-L. Verdier, Des catégories dérivées des catégories abéliennes, Astérisque (1996), no. 239, xii+253 pp. (1997), with a preface by Luc Illusie, edited and with a note by Georges Maltsiniotis. MR 1453167 (98c:18007)

Mohamed Barakat barakat@mathematik.uni-kl.de

Department of Mathematics, University of Kaiserslautern, 67653 Kaiserslautern, Germany

Markus Lange-Hegermann markus.lange.hegermann@rwth-aachen.de

Lehrstuhl B für Mathematik, RWTH Aachen University, 52062 Aachen, Germany 